\title{
Inter-study reproducibility of cardiovascular magnetic resonance myocardial feature tracking
}

\author{
Geraint Morton ${ }^{1 \dagger}$, Andreas Schuster $^{1 \dagger}$, Roy Jogiya ${ }^{1}$, Shelby Kutty ${ }^{2}$, Philipp Beerbaum ${ }^{3}$ and Eike Nagel ${ }^{1 *}$
}

\begin{abstract}
Background: Cardiovascular magnetic resonance myocardial feature tracking (CMR-FT) is a recently described method of post processing routine cine acquisitions which aims to provide quantitative measurements of circumferentially and radially directed ventricular wall strain. Inter-study reproducibility is important for serial assessments however has not been defined for CMR-FT.
\end{abstract}

Methods: 16 healthy volunteers were imaged 3 times within a single day. The first examination was performed at 0900 after fasting and was immediately followed by the second. The third, non-fasting scan, was performed at 1400.

CMR-FT measures of segmental and global strain parameters were calculated. Left ventricular (LV) circumferential and radial strain were determined in the short axis orientation (ECCSAX and $\operatorname{Err}_{S A X}$ respectively). LV and right ventricular longitudinal strain and LV radial strain were determined from the 4-chamber orientation $\left(E\left\|_{L V}, E\right\|_{R V}\right.$, and Err LAX respectively). LV volumes and function were also analysed.

Inter-study reproducibility and study sample sizes required to demonstrate $5 \%$ changes in absolute strain were determined by comparison of the first and second exams. The third exam was used to determine whether diurnal variation affected reproducibility.

Results: CMR-FT strain analysis inter-study reproducibility was variable. Global strain assessment was more reproducible than segmental analysis. Overall $\mathrm{ECC}_{\mathrm{SAX}}$ was the most reproducible measure of strain: coefficient of variation (CV) 38\% and 20.3\% and intraclass correlation coefficient (ICC) 0.68 (0.55-0.78) and 0.7 (0.32-0.89) for segmental and global analysis respectively. The least reproducible segmental measure was E $\|_{R V}$ : CV $60 \%$ and ICC 0.56 (0.41-0.69) whilst the least reproducible global measure was Err_AX: CV 33.3\% and ICC 0.44 (0-0.77). Variable reproducibility was also reflected in the calculated sample sizes, which ranged from 11 (global Ecc SAX) $_{\text {) }} 156$ subjects (segmental E $\|_{\mathrm{RV}}$ ). The reproducibility of LV volumes and function was excellent. There was no diurnal variation in global strain or LV volumetric measurements.

Conclusions: Inter-study reproducibility of CMR-FT varied between different parameters, as summarized above and was better for global rather than segmental analysis. It was not measurably affected by diurnal variation. CMR-FT may have potential for quantitative wall motion analysis with applications in patient management and clinical trials. However, inter-study reproducibility was relatively poor for segmental and long axis analyses of strain, which have yet to be validated, and may benefit from further development.

\footnotetext{
* Correspondence: EN: eike.nagel@kcl.ac.uk

${ }^{\dagger}$ Equal contributors

'King's College London British Heart Foundation (BHF) Centre of Excellence;

National Institute of Health Research (NIHR) Biomedical Research Centre at

Guy's and St. Thomas' NHS Foundation Trust; Wellcome Trust and

Engineering and Physical Sciences Research Council (EPSRC) Medical

Engineering Centre; Division of Imaging Sciences and Biomedical

Engineering, The Rayne Institute, 4th Floor Lambeth Wing, St. Thomas'

Hospital, London SE1 7EH, United Kingdom

Full list of author information is available at the end of the article
} 


\section{Background}

Myocardial wall motion analysis is key for assessment of left ventricular (LV) and right ventricular (RV) function. It is important for the evaluation of cardiomyopathies [1] and for identification of hibernation and ischaemia during dobutamine stress in patients with coronary artery disease (CAD) [2]. Cardiovascular magnetic resonance (CMR) is an established method for wall motion assessment as it provides excellent endocardial border definition and can be combined with other techniques for a comprehensive, radiation-free, cardiac assessment in a single session [3]. Currently visual assessment of wall motion is standard however this is limited by inter-observer variability [4]. To date quantitative techniques for assessment of wall motion, such as myocardial tagging, have not been widely adopted due to the requirement to perform additional scans and the associated complex and time-consuming post-processing $[5,6]$. Alternative methods, which allow robust and user-independent quantitative wall motion assessment, are therefore desirable.

CMR myocardial feature-tracking (CMR-FT) has been recently described $[7,8]$ and is a method of quantitative wall motion assessment which is analogous to echocardiographic speckle-tracking. Standard CMR cine images are analysed off-line and acquisition of additional sequences is therefore not required. CMR-FT aims to provide measurements of circumferentially, radially and longitudinally directed ventricular wall strain. However, in order to be robust and useful, analytical techniques must be sufficiently reproducible. Reasonable inter and intra-observer reproducibility of feature tracking has already been demonstrated $[9,10]$. The aim of this study therefore was to evaluate the inter-study reproducibility of CMR-FT and ascertain whether this is affected by detectable physiological variation.

\section{Methods \\ Population}

16 healthy volunteers were recruited by emailing university staff and students. Exclusion criteria were: known cardiac, respiratory or renal disease or a contraindication to MRI. The local ethics committee approved the study and all participants gave written informed consent.

\section{Data acquisition}

Participants underwent 3 CMR examinations on the same day. All imaging was performed on a 3 Tesla clinical MRI scanner (Achieva, Philips Medical Systems, Best, The Netherlands), with participants supine, and using a 32-channel phased array receiver cardiac coil. On the day of the scans subjects were asked to fast from midnight. The first CMR examination (exam A) was performed at 0900, and the second (exam B) immediately afterwards at 0930. In order to try and maximise physiological changes participants then left the department and ate and drank as normal. They returned at 1400 for the third scan (exam C). Exams A and B were acquired to assess for the inherent variability of CMRFT and exams A and $\mathrm{C}$ to assess for additional physiological changes due to circadian rhythms, and changes in hydration.

As part of the protocol scans included an initial survey, coil reference scan and planning to define imaging planes. Cine images were acquired using a standard balanced steady state free precession (SSFP) sequence. Planning and acquisition were as follows: A pseudo 2chamber cine was planned from the axial survey images and this was subsequently used to plan a pseudo 4chamber cine. Systolic frames from the pseudo 2 and 4chamber cines were then used to plan 3 short axis cine slices of the basal, mid and apical LV. Diastolic frames from preceding cines were then used to plan definitive views. The pseudo 2-chamber and short axis views were used to plan the 4-chamber cine. This 4-chamber view and the pseudo short axis slices were used to plan the 2chamber cine. The 2 and 4-chamber views were used to plan, contiguous short axis (SA) cine slices covering the entire LV, and the 4-chamber and short axis views to plan a 3-chamber cine. SSFP cine in-plane resolution was $1.8 \times 2 \mathrm{~mm}$ and slice thickness $8 \mathrm{~mm}$. The protocol was the same for all 3 scans and for all participants. Participants were removed from the scanner between each exam. Each scan lasted approximately 30-minutes.

\section{Data analysis}

\section{Feature tracking}

Images were analysed in a random order by a blinded experienced observer. CMR-FT strain analysis was performed using dedicated prototype software (Diogenes MRI, Tomtec, Unterschleissheim, Germany). Endocardial contours were manually drawn in all views included in the analysis. The 4-chamber view was used to determine RV and LV longitudinal strain and LV radial strain $\left(\mathrm{Ell}_{\mathrm{RV}}\right.$ and Ell $_{\text {LV }}$ and $\left.\operatorname{Err}_{\mathrm{LAX}}\right)$. LV short axis circumferential (EcCSAX) and radial strains $\left(\mathrm{Err}_{\mathrm{SAX}}\right)$ were calculated from a midventricular short-axis view containing both papillary muscles. The RV upper septal insertion point was manually defined to allow accurate segmentation according to a recognized standard model [11]. All parameters were analysed for all three exams. Strain values (\% change from baseline) were obtained for each segment and global values defined as the mean of all segmental values.

\section{Left ventricular volumes and function}

Images were analysed in a random order by a blinded experienced observer using CMR42 (Circle, Calgary, Canada). The mitral valve plane and apex were identified from the 4-chamber view in end-diastole and end- 
systole. The LV endocardial border was automatically defined in the corresponding end-systolic and enddiastolic SA slices and was manually corrected where required. Papillary muscles were excluded.

\section{Statistical analysis}

Statistical analysis was performed using Microsoft Excel and IBM SPSS Statistics version 19. For inter-study reproducibility evaluation a coefficient of variation (CV), intraclass correlation coefficient (ICC) and Bland Altman plots were calculated. For each parameter the mean value and differences between exam $\mathrm{A}$ and $\mathrm{B}$ and exam $\mathrm{A}$ and $\mathrm{C}$ values were calculated. The $\mathrm{CV}$ was defined as the standard deviation of the differences divided by the mean [12]. Analyses were all performed on a per observation basis.

The significance of differences in reproducibility between exam A and B (reproducibility $\mathrm{AB}$ ) and exam $\mathrm{A}$ and $\mathrm{C}$ (reproducibility $\mathrm{AC}$ ) was evaluated by comparing the squared differences between the observations. These were compared directly with a paired $t$ test if normally distributed (LV volumes and function) or after natural $\log$ transformation if not normally distributed (strain) [12]. The Shapiro-Wilk test determined normality. The mean values for each parameter from exam $A, B$ and $C$ were not normally distributed and therefore were compared using the Friedman test.

Study sample sizes required to show a 5\% absolute change in strain with a power of $90 \%$ and an $\alpha$ error of 0.05 were calculated as follows [12]:

$$
n=f(\alpha, \mathrm{P}) \cdot \sigma^{2} \cdot{ }^{2} / \delta^{2}
$$

where $\mathrm{n}$ is the sample size, $\mathrm{f}=10.5$ for $\alpha 0.05$ and $\mathrm{P} 0.9$, $\sigma$ the inter-study standard deviation and $\delta$ the magnitude of the difference to be detected.

Continuous data are presented as mean \pm standard deviation. Significance was determined as $\mathrm{p}<0.05$.

\section{Results}

\section{Participant details}

Participant age was $27.9 \pm 5.7$ and body mass index $26.2 \pm 6.8$. Eight were male and 8 female. One participant chose not to re-attend for scan C after scans A and B. All other participants completed the protocol. The CMR data of one other participant was found to be incompatible with the CMR-FT software (transient high signal in the pulmonary arteries affected the grey scaling obscuring endocardial border tracking). This participant was therefore only included in the volumetric assessments. Image quality was otherwise good or excellent in all subjects.

\section{Feature tracking}

Mean segmental and global strain parameters for all three exams are summarised in Table 1. Mean segmental
Table 1 Mean \pm standard deviation segmental and global strain values for the combined study population

\begin{tabular}{|c|c|c|c|c|}
\hline & \multicolumn{3}{|c|}{ Exam } & \multirow[t]{2}{*}{$p$} \\
\hline & A & B & C & \\
\hline \multicolumn{5}{|c|}{ Segmental } \\
\hline $\operatorname{Err}_{\text {SAX }}$ & $22.4 \pm 9.6$ & $19.2 \pm 8.0$ & $20.9 \pm 9.0$ & 0.04 \\
\hline $\mathrm{ECC}_{\mathrm{SAX}}$ & $-17.6 \pm 8.1$ & $-16.6 \pm 8.4$ & $-18.2 \pm 8.9$ & 0.06 \\
\hline $\operatorname{Err}_{\text {LAX }}$ & $16.2 \pm 10.2$ & $17.7 \pm 10.3$ & $17.9 \pm 10.5$ & 0.25 \\
\hline$E \|_{L V}$ & $-21.0 \pm 11.0$ & $-20.0 \pm 11.0$ & $-19.2 \pm 12.8$ & 0.23 \\
\hline$E \|_{R V}$ & $-23.7 \pm 16.4$ & $-21.7 \pm 13.5$ & $-20.6 \pm 14.7$ & 0.25 \\
\hline \multicolumn{5}{|l|}{ Global } \\
\hline $\operatorname{Err}_{\text {SAX }}$ & $22.6 \pm 7.9$ & $19.4 \pm 6.7$ & $20.5 \pm 5.2$ & 0.40 \\
\hline ECC SAX $_{\text {SAX }}$ & $-17.6 \pm 5.0$ & $-16.6 \pm 4.4$ & $-18.1 \pm 4.3$ & 0.81 \\
\hline Err $_{\text {LAX }}$ & $16.2 \pm 5.6$ & $17.7 \pm 5.6$ & $17.9 \pm 3.8$ & 0.29 \\
\hline$E \|_{L V}$ & $-21.0 \pm 5.1$ & $-20.0 \pm 5.3$ & $-19.2 \pm 5.3$ & 0.06 \\
\hline$E \|_{R V}$ & $-23.8 \pm 9.9$ & $-21.8 \pm 5.7$ & $-19.1 \pm 8.7$ & 0.26 \\
\hline
\end{tabular}

Values are in \%. $p$ values refer to the significance of differences between the mean values from exams $A, B$ and $C$. *denotes significant differences.

$\operatorname{Err}_{\text {SAX }}$ was significantly different $(\mathrm{p}=0.04)$ between the scans although the largest difference was between scans $A$ and B. There were no significant differences in any of the other strain parameters.

\section{Inter-study reproducibility}

$\mathrm{CV}$, ICCs and sample size calculations for each strain parameter are summarised in Table 2. Reproducibility

Table 2 Segmental and global inter-study reproducibility for five different feature tracking strain parameters and the study sample size required to detect an absolute change of $5 \%$ with $90 \%$ power and an a error of 0.05

\begin{tabular}{|c|c|c|c|c|}
\hline & $\begin{array}{c}\text { Mean } \\
\text { difference } \pm \text { SD }\end{array}$ & $\begin{array}{l}\text { Coefficient } \\
\text { of Variation }\end{array}$ & $\begin{array}{c}\text { ICC } \\
(95 \% \mathrm{Cl})\end{array}$ & $\begin{array}{l}\text { Sample } \\
\text { size }\end{array}$ \\
\hline \multicolumn{5}{|c|}{ Segmental } \\
\hline ErrsAX $_{\text {SAX }}$ & $-3.5 \pm 7.5 \%$ & $35.9 \%$ & $0.57(0.35-0.71)$ & 48 \\
\hline $\mathrm{ECC}_{\mathrm{SAX}}$ & $1.2 \pm 6.5 \%$ & $38.0 \%$ & $0.68(0.55-0.78)$ & 36 \\
\hline Err $_{\text {LAX }}$ & $1.5 \pm 9.2 \%$ & $53.2 \%$ & $0.62(0.47-0.73)$ & 72 \\
\hline$E \|_{L V}$ & $-1.0 \pm 11.3$ & $55.4 \%$ & $0.59(0.44-0.71)$ & 108 \\
\hline$E \|_{R V}$ & $-1.9 \pm 13.6$ & $60.0 \%$ & $0.56(0.41-0.69)$ & 156 \\
\hline \multicolumn{5}{|l|}{ Global } \\
\hline Err $_{\text {SAX }}$ & $-3.2 \pm 5.7 \%$ & $27.2 \%$ & $0.61(0.15-0.85)$ & 28 \\
\hline $\mathrm{ECC}_{S A X}$ & $1.0 \pm 3.5 \%$ & $20.3 \%$ & $0.70(0.32-0.89)$ & 11 \\
\hline Err $_{\text {LAX }}$ & $1.5 \pm 5.8 \%$ & $33.3 \%$ & $0.44(0-0.77)$ & 29 \\
\hline$E \|_{L V}$ & $-1.08 \pm 5.4 \%$ & $26.4 \%$ & $0.44(0-0.77)$ & 25 \\
\hline$E \|_{R V}$ & $-1.9 \pm 6.8 \%$ & $29.9 \%$ & $0.62(0.20-0.85)$ & 39 \\
\hline
\end{tabular}

$S D=$ Standard deviation; $I C C=$ Intraclass correlation coefficient; $C l=$ Confidence Interval

Sample size numbers must be doubled for studies comparing active treatment vs. placebo. 

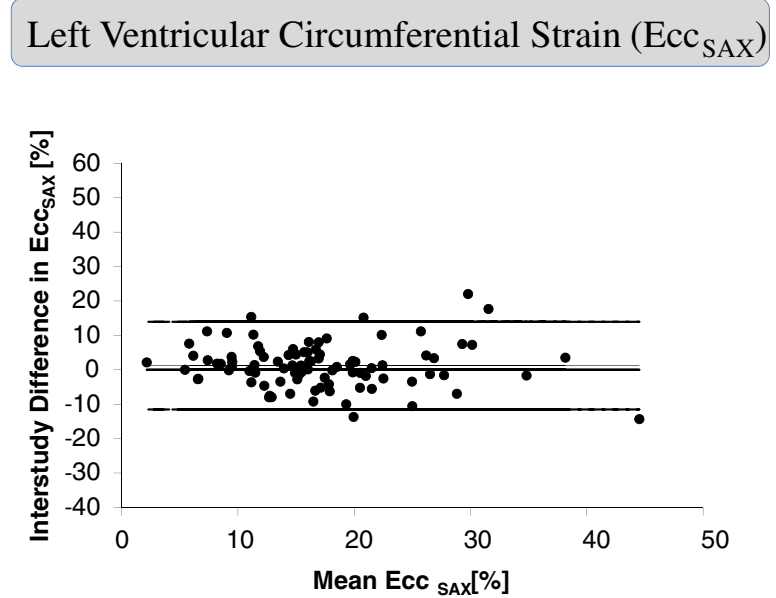

Right Ventricular Longitudinal Strain $\left(\mathrm{Ell}_{\mathrm{RV}}\right)$

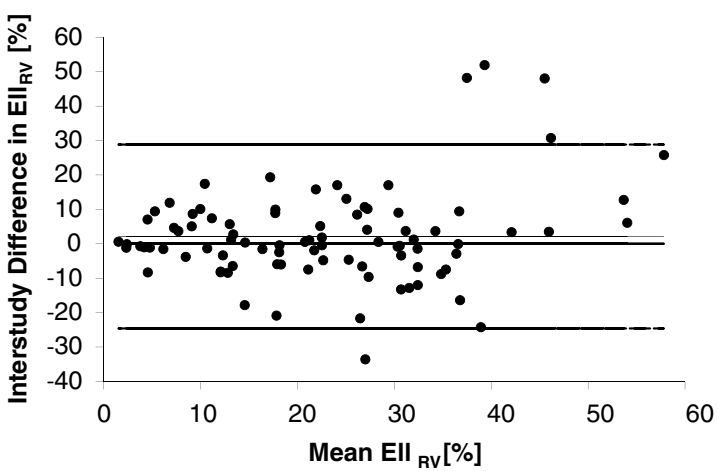

Figure 1 Inter-study agreement of segmental strain as determined by feature tracking. Bland Altman plots with limits of agreement (95\% confidence intervals) demonstrating the inter-study reproducibility of CMR myocardial feature tracking segmental left ventricular circumferential and right ventricular longitudinal strain.

was highly variable. Global strain assessment was more reproducible than segmental analysis. Overall Ecc SAX was the most reproducible measure of strain: CV 38\% and $20.3 \%$ and ICC $0.68(0.55-0.78)$ and 0.7 (0.32-0.89) for segmental and global analysis respectively. The least reproducible segmental measure was $\mathrm{Ell}_{\mathrm{RV}}$ : CV 60\% and ICC 0.56 (0.41-0.69) whilst the least reproducible global measure was $\operatorname{Err}_{\text {LAX: }}$ CV 33.3\% and ICC 0.44 (0-0.77). Bland-Altman plots demonstrate the reproducibility of segmental $\mathrm{ECC}_{\mathrm{SAX}}$ and $\mathrm{Ell}_{\mathrm{RV}}$ (Figure 1).

This variable reproducibility is reflected in the sample size calculations. 5\% changes in absolute global strain can be detected in follow-up studies with relatively few patients whilst many more are required for some segmental strain parameters. Sample sizes for global strain parameters ranged from $11\left(\mathrm{EcC}_{\mathrm{SAX}}\right)$ to $39\left(\mathrm{Ell}_{\mathrm{RV}}\right)$ and for segmental strain from $36\left(\mathrm{EcC}_{\mathrm{SAX}}\right)$ to $156\left(\mathrm{Ell}_{\mathrm{RV}}\right)$.

\section{Comparison of inter-study reproducibility $A B$ with $A C$}

Inter-study reproducibility $A C$ was not significantly different from reproducibility $\mathrm{AB}$ for any segmental or global parameters ( $p>0.05$ for all).

Table 3 Mean left ventricular volumes and function for the entire study population

\begin{tabular}{|c|c|c|c|c|}
\hline & \multicolumn{3}{|c|}{ Exam } & \multirow[t]{2}{*}{$p$} \\
\hline & A & B & C & \\
\hline LVEDV & $161.7 \pm 33.3 \mathrm{ml}$ & $162.5 \pm 37.0 \mathrm{ml}$ & $161.2 \pm 39.7 \mathrm{ml}$ & 0.76 \\
\hline LVESV & $67.5 \pm 17.3 \mathrm{ml}$ & $68.9 \pm 20.0 \mathrm{ml}$ & $64.9 \pm 18.3 \mathrm{ml}$ & 0.28 \\
\hline LVEF & $58.5 \pm 3.2 \%$ & $58.0 \pm 4.3 \%$ & $59.6 \pm 4.5 \%$ & 0.86 \\
\hline
\end{tabular}

$p$ values refer to the significance of differences between exam $A, B$ and $C$ $\angle V E D V=$ left ventricular end-diastolic volume; $L V E S V=$ left ventricular endsystolic volume; $L V E F=$ left ventricular ejection fraction.

\section{LV volumes and function} Inter-study reproducibility

Mean LV volumes and function are shown in Table 3. There were no significant differences in LV volumes or function between the three exams. Inter-study reproducibility was excellent overall. CV and ICC for LV enddiastolic volume, end-systolic volume and ejection fraction were $4.2 \%$ and $0.98 ; 8.3 \%$ and 0.95 ; and $4.6 \%$ and 0.75 respectively (Table 4 ).

\section{Comparison of inter-study reproducibility $A B$ with $A C$}

There were no significant differences between reproducibility $\mathrm{AB}$ and $\mathrm{AC}$ for $\mathrm{LV}$ volumes or function $(\mathrm{p}>0.05$ for all).

\section{Discussion}

This study demonstrates that the inter-study reproducibility of CMR-FT parameters is highly variable and is better for global rather than segmental measures. Ecc SAX $_{\text {S }}$ is the most reproducible whilst others such as Ell $\mathrm{RV}_{\mathrm{S}}$ and $\operatorname{Err}_{\text {LAX }}$ are less reproducible. Furthermore there was no measurable diurnal variation.

Table 4 Inter-study reproducibility for left ventricular volumes and function

\begin{tabular}{lccc}
\hline difference \pm SD & $\begin{array}{c}\text { Coefficient of } \\
\text { Variation }\end{array}$ & $\begin{array}{c}\text { Intraclass correlation } \\
\text { coefficient (95\%Cl) }\end{array}$ \\
\hline LVEDV & $-0.4 \pm 6.9 \mathrm{ml}$ & $4.2 \%$ & $0.98(0.97-1.0)$ \\
LVESV & $-0.8 \pm 5.3 \mathrm{ml}$ & $8.3 \%$ & $0.95(0.88-0.98)$ \\
LVEF & $0.3 \pm 2.6 \mathrm{ml}$ & $4.6 \%$ & $0.75(0.43-0.91)$ \\
\hline
\end{tabular}

$\angle V E D V=$ left ventricular end-diastolic volume; $L V E S V=$ left ventricular endsystolic volume; $L V E F=$ left ventricular ejection fraction; $C l=$ confidence interval. 


\section{CMR feature tracking}

Abnormalities or inter-study changes in myocardial strain can occur without any detectable changes in LV volumes or function. This has been demonstrated, for example, in studies examining the treatment effect of stem cells in myocardial infarction [13,14]. Myocardial strain imaging may therefore allow early detection of abnormalities and subsequently allow early diagnosis and intervention. However despite this imaging techniques for strain analysis have not been widely adopted. CMRFT has been described in detail previously [8] and appears to be a promising new tool for quantitative assessment of wall motion analysis. Borders within the image, such as the border between the LV endocardium and LV blood, are identified and then tracked from frame to frame. This relies on quantification of changes in signal intensity within voxels from one frame to the next. Frame-to-frame displacement of these signal changes is used to calculate local myocardial velocity and deformation or strain.

The accuracy of CMR-FT is likely to depend on the orientations and dimensions of the relevant border or features relative to voxels of the slice. Furthermore CMR- FT software derives strain measurements from the apparent in-plane movements. However the apparent movements of features in 2D cine images may be caused by throughplane displacements of oblique or tapering structures, and not only by in-plane movements. This potential limitation of the technique may explain some of the variability seen in reproducibility in this study.

Global Ecc $\mathrm{SAX}_{\mathrm{SAX}}$ from the mid LV slice has been shown to correlate strongly with myocardial tagging using Harmonic Phase Imaging (HARP) in patients with Duchene Muscular Dystrophy and varying LV dysfunction and also in normal volunteers [15]. Ecc $\mathrm{SAx}_{\mathrm{SA}}$ has also been compared with a complementary spatial modulation of magnetization (CSPAMM) myocardial tagging reference method in a subsequent study [16]; agreement was modest and notably segmental strain measurements were less good than global Ecc $\mathrm{SAX}_{\mathrm{X}}$ measurements. However, to date, other CMR-FT parameters have not been validated against a reference standard for the evaluation of myocardial strain. Further validation of CMR-FT strain measurements, particularly segmental measurements, is therefore desirable.

\section{Inter-study reproducibility}

Inter-study reproducibility of a technique is key where repeated examinations are required. The absence of ionising radiation means that CMR lends itself well to repeated testing. Wall motion assessment with CMR-FT could therefore potentially be useful for serial examinations of ventricular function to assess disease progression, treatment efficacy or determine the timing of therapy. Serial examinations are important in a multitude of clinical conditions and also form a key component of many studies. Higher reproducibility means that smaller changes can be detected with increased reliability. This also results in cost-efficiencies, as fewer subjects are required in clinical trials [17].

Our sample size calculations demonstrate that relatively small sample sizes are required to detect 5\% changes in global strain parameters, particularly Ecc $\mathrm{CAX}_{\mathrm{SAX}}$. However, a $5 \%$ change might be a relatively large effect, and larger sample sizes would be required if subtle changes in strain were likely to be clinically relevant. Furthermore, lower reproducibility of some segmental strain parameters means that large sample sizes would be required to detect differences in these. This is likely to be particularly relevant for assessing patients with CAD when regional analysis is crucial. Therefore whilst this new technique is promising for quantitative wall motion assessment its utility would be greater if further method developments improved reproducibility.

To our knowledge this is the first assessment of CMRFT inter-study reproducibility. However, previous studies by our group have suggested that inter and intra observer reproducibility is higher for $\mathrm{Ecc}_{\mathrm{SAX}}$ than for $\operatorname{Err}_{\mathrm{SAX}}$ both in volunteers and in patients with ischaemic cardiomyopathy $[9,10]$. Inter-study variability was of a similar magnitude to the intra-observer and inter-observer variability reported previously although intra and interobserver reproducibility may be superior to inter-study reproducibility.

This study included normal volunteers and image quality was good to excellent in all cases. Reproducibility and sample sizes may therefore be different in patient studies or when image quality is reduced. However, in previous studies of volunteers [9] and patients with ischaemic cardiomyopathy [10], CMR-FT intra- and inter-observer reproducibility were no worse during low-dose dobutamine infusion than at rest, even though dobutamine is often associated with a deterioration in image quality. Furthermore, CMR-FT reproducibility in patients with ischemic cardiomyopathy appeared to be comparable to that of healthy volunteers despite reduced LV function in the patients. These observations suggest that the findings of this study can be applied to patients. However, it is important to note that in the patient study $13 \%$ of segments were excluded, mainly due to breathing artefacts.

This study also confirms the excellent inter-study reproducibility of CMR for evaluating LV volumes and function demonstrated in previous studies [12,17]. In keeping with these studies, using $\mathrm{CV}$ as a measure of inter-study reproducibility, we also found LV endsystolic volume to be slightly less reproducible than both LV end-diastolic volume and LV ejection fraction. However, ICC was lower for ejection fraction than for LV 
volumes, emphasizing the fact that multiple measures of inter-study reproducibility exist and the potential difficulties in comparing results from studies using different statistical methods. We have reported multiple measures of reproducibility in order to be comprehensive.

\section{Physiological variation}

Many biological processes exhibit circadian rhythms and heart rate and blood pressure changes are well known [18]. Consequently studies are often designed to minimise the effects of physiological variation either as a result of circadian rhythms or changes in participants' volume status and therefore ventricular volumes [12]. However, data on whether detectable diurnal changes in left ventricular volumes and function actually occur are limited $[19,20]$. Previous echocardiographic studies have suggested that there are no diurnal variations in LV volumes and systolic function. Nonetheless variations in some parameters, of up to approximately $20 \%$, have been demonstrated in healthy volunteers $[19,21]$. In keeping with these studies we did not detect any diurnal changes in LV volumes and function or in LV or RV myocardial strain. Furthermore, the inter-study reproducibility of LV volume and function and strain measurements was not measurably affected by diurnal variation. These findings suggest that diurnal variations are not currently important considerations when repeated studies are performed for CMR-FT and/or volumetric analysis. However, it is important to note that the higher variability of the strain measurements means that small changes are unlikely to have been detected. The sample size calculations suggest that our sample was large enough to detect a $5 \%$ change in $\mathrm{Ecc}_{\mathrm{SAX}}$.

\section{Comparison with speckle tracking}

Speckle tracking with echocardiography is a more established technique than CMR-FT. [22] Previous echocardiography based studies have shown the clinical utility of speckle tracking for LV viability assessment and planning of revascularisation, assessment of patients with acute myocardial infarction and heart failure with normal ejection fraction and as an early marker of LV dysfunction in patients with Tetralogy of Fallot [23-27]. Data on the inter-study reproducibility of strain analysis speckle tracking is limited and it is therefore difficult to draw comparisons with the CMR findings of this study. Reasonable inter-study reproducibility of left ventricular rotation parameters (as opposed to strain) has been demonstrated [28] using different measures of reproducibility.

Recently echocardiographic global longitudinal speckle strain was shown to be an independent predictor of mortality in a population of 546 consecutive patients undergoing echocardiography for left ventricular assessment [29]. Global longitudinal strain was a stronger predictor than both ejection fraction and wall motion scoring. This raises the possibility that the analogous global longitudinal LV feature tracking strain measured in this study, and possibly other strain parameters, may also be useful prognostic markers and our data show reasonably good reproducibility of global parameters. Larger scale long-term CMR studies are required to test this hypothesis.

Comparative studies between CMR-FT and speckle tracking have not yet been performed. Both techniques have potential advantages. Reliable endocardial border detection is key for strain imaging and superior image quality and endocardial border definition with CMR may be an advantage. Echocardiography has different axial and lateral resolutions and can also be affected by limited acoustic windows. Border tracking may therefore be more reliable with better image quality. Conversely although CMR provides relatively good contrast to noise at most epicardial and endocardial boundaries it may not be able to detect similar intra-myocardial features, and therefore intra-myocardial strain, as well as speckle tracking. CMR myocardial tagging imaging techniques may also be superior to CMR-FT in this respect. Moreover, echocardiography has superior temporal resolution, which may translate into an advantage for speckle tracking over CMR-FT.

\section{Limitations}

The main limitations of this study are its small size and the inclusion of normal volunteers rather than patients. However it is common to evaluate emerging techniques in volunteers initially and these studies are important platforms for further method improvement and subsequent patient studies. Moreover limited previously published data suggest that volunteer and patient reproducibility may well be similar as discussed above.

\section{Conclusions}

Inter-study reproducibility of CMR-FT is highly variable. At present reproducibility is best for global measurements and LV circumferential strain is the most reproducible strain parameter. CMR-FT strain analysis is not measurably influenced by diurnal variation, although this may be due to the small sample size. CMR myocardial strain analysis with feature tracking may have potential clinical and research applications. However the technique, particularly segmental and long axis analyses of strain, would benefit from improved inter-study reproducibility and also further validation studies.

\section{Competing interests}

The authors declare that they have no competing interests.

\section{Acknowledgments}

The authors thank Siobhan Crighton of King's College London for her assistance with the statistical analysis. 
This work was supported by a European Union Grant (Grant number 224495); the British Heart Foundation (Research Excellence Award RE/08/003 and FS/10/029/28253); the Biomedical Research Centre (grant number BRCCTF 196) and the Wellcome Trust and EPSRC (grant number WT 088641/Z/ 09/Z).

\section{Author details}

${ }^{1}$ King's College London British Heart Foundation (BHF) Centre of Excellence: National Institute of Health Research (NIHR) Biomedical Research Centre at Guy's and St. Thomas' NHS Foundation Trust; Wellcome Trust and Engineering and Physical Sciences Research Council (EPSRC) Medical Engineering Centre; Division of Imaging Sciences and Biomedical Engineering, The Rayne Institute, 4th Floor Lambeth Wing, St. Thomas' Hospital, London SE1 7EH, United Kingdom. ${ }^{2}$ Joint Division of Pediatric Cardiology, University of Nebraska College of Medicine/ Creighton University School of Medicine, Children's Hospital and Medical Center, Omaha, NE, USA. ${ }^{3}$ Departments for Radiology and Paediatric Cardiology, St Radboud Medical University, Nijmegen, The Netherlands.

\section{Authors' contributions}

GM and AS designed the study protocol, acquired and analysed the data and drafted the manuscript. RJ, SK and PB helped acquire and analyse the data and critically revised the manuscript. EN assisted with study design and interpretation of data and critically revised the manuscript. All authors read and approved the final manuscript.

Received: 5 December 2011 Accepted: 21 June 2012

Published: 21 June 2012

\section{References}

1. Karamitsos TD, Francis JM, Myerson S, Selvanayagam JB, Neubauer S: The role of cardiovascular magnetic resonance imaging in heart failure. J Am Coll Cardiol 2009, 54(15):1407-1424.

2. Schuster A, Morton G, Chiribiri A, Perera D, Vanoverschelde J, Nagel E: Imaging in the management of ischemic cardiomyopathy: special focus on magnetic resonance. J Am Coll Cardiol 2012, 59(4):359-70.

3. Morton G, Schuster A, Perera D, Nagel E: Cardiac magnetic resonance imaging to guide complex revascularization in stable coronary artery disease. Eur Heart J 2010, 31:2209-2215.

4. Paetsch I, Jahnke C, Ferrari VA, Rademakers FE, Pellikka PA, Hundley WG, Poldermans D, Bax JJ, Wegscheider K, Fleck E, Nagel E: Determination of interobserver variability for identifying inducible left ventricular wall motion abnormalities during dobutamine stress magnetic resonance imaging. Eur Heart J 2006, 27(12):1459-1464.

5. Ibrahim E-SH: Myocardial tagging by Cardiovascular Magnetic Resonance: evolution of techniques-pulse sequences, analysis algorithms, and applications. J Cardiovasc Magn Reson 2032, 13(1):36.

6. Schuster A, Nagel E: Toward Full Quantification of Wall Motion with MRI. Curr Cardiovasc Imaging Reports 2011, 4:85-86.

7. Maret E, Todt T, Brudin L, Nylander E, Swahn E, Ohlsson JL, Engvall JE: Functional measurements based on feature tracking of cine magnetic resonance images identify left ventricular segments with myocardial scar. Cardiovasc Ultrasound 2009, 7:53.

8. Hor KN, Baumann R, Pedrizzetti G, Tonti G, Gottliebson WM, Taylor M, Benson W, Mazur W: Magnetic resonance derived myocardial strain assessment using feature tracking. J Vis Exp 2011, 48.

9. Schuster A, Kutty S, Padiyath A, Parish V, Gribben P, Danford DA, Makowski MR, Bigalke B, Beerbaum P, Nagel E: Cardiovascular Magnetic Resonance Myocardial feature tracking detects Quantitative Wall Motion during Dobutamine Stress. Journal of Cardiovascular Magnetic Resonance: Official Journal Of The Society for Cardiovascular Magnetic Resonance 2011, 13(1):58.

10. Schuster A, Paul M, Bettencourt N, Morton G, Chiribiri A, Ishida M, Hussain S, Jogiya R, Kutty S, Bigalke B, et al: Cardiovascular magnetic resonance myocardial feature tracking for quantitative viability assessment in ischemic cardiomyopathy. Int I Cardiol 2011, doi:10.1016/.jijcard.2011.10.137 [E-pub ahead of print].

11. Cerqueira MD, Weissman NJ, Dilsizian V, Jacobs AK, Kaul S, Laskey WK, Pennell DJ, Rumberger JA, Ryan T, Verani MS, Imaging AHAWGoMSaRfC: Standardized myocardial segmentation and nomenclature for tomographic imaging of the heart: a statement for healthcare professionals from the Cardiac Imaging Committee of the Council on Clinical Cardiology of the American Heart Association. Circulation 2002, 105(4):539-542.

12. Grothues F, Smith GC, Moon JCC, Bellenger NG, Collins P, Klein HU, Pennell DJ: Comparison of interstudy reproducibility of cardiovascular magnetic resonance with two-dimensional echocardiography in normal subjects and in patients with heart failure or left ventricular hypertrophy. Am J Cardiol 2002, 90(1):29-34.

13. Hopp E, Lunde K, Solheim S, Aakhus S, Arnesen H, Forfang K, Edvardsen T, Smith $\mathrm{H}-\mathrm{J}$ : Regional myocardial function after intracoronary bone marrow cell injection in reperfused anterior wall infarction - a cardiovascular magnetic resonance tagging study. Journal of cardiovascular magnetic resonance: official journal of the Society for Cardiovascular Magnetic Resonance 2011, 13:22.

14. Herbots L, D'hooge J, Eroglu E, Thijs D, Ganame J, Claus P, Dubois C, Theunissen K, Bogaert J, Dens J, et al: Improved regional function after autologous bone marrow-derived stem cell transfer in patients with acute myocardial infarction: a randomized, double-blind strain rate imaging study. Eur Heart J 2008, 30(6):662-670.

15. Hor KN, Gottliebson WM, Carson C, Wash E, Cnota J, Fleck R, Wansapura J, Klimeczek P, Al-Khalidi HR, Chung ES, et al: Comparison of magnetic resonance feature tracking for strain calculation with harmonic phase imaging analysis. JACC Cardiovasc Imaging 2010, 3:144-151.

16. Harrild DM, Han Y, Geva T, Zhou J, Marcus E, Powell AJ: Comparison of cardiac MRI tissue tracking and myocardial tagging for assessment of regional ventricular strain. The international journal of cardiovascular imaging 2012. http://www.springerlink.com/content/y3402gt|22648666/

17. Bellenger $N$, Davies $L$, Francis J, Coats A, Pennell D: Reduction in Sample Size for Studies of Remodeling in Heart Failure by the Use of Cardiovascular Magnetic Resonance. J of Cardiovascular Magnetic Resonance 2000, 2(4):271-278

18. Pickering TG, Harshfield GA, Kleinert HD, Blank S, Laragh JH: Blood pressure during normal daily activities, sleep, and exercise. Comparison of values in normal and hypertensive subjects. JAMA 1982, 247(7):992-996.

19. Karabag T, Aydin M, Dogan SM, Sayin MR, Cetiner MA: The Influence of Circadian Variations on Echocardiographic Parameters in Healthy People. Echocardiography 2011, 28(6):612-618.

20. Bull SC, Main ML, Stevens GR, Goldman JH, Constable SA, Becher H: Cardiac Toxicity Screening by Echocardiography in Healthy Volunteers: A Study of the Effects of Diurnal Variation and Use of a Core Laboratory on the Reproducibility of Left Ventricular Function Measurement. Echocardiography 2011, 28(5):502-507.

21. Voutilainen S, Kupari M, Hippelainen M, Karppinen K, Ventila M: Circadian variation of left ventricular diastolic function in healthy people. Heart 1996, 75(1):35-39.

22. Pavlopoulos $\mathrm{H}$, Nihoyannopoulos $\mathrm{P}$ : Strain and strain rate deformation parameters: from tissue Doppler to 2D speckle tracking. Int I Cardiovasc Imaging 2008, 24(5):479-491.

23. Bansal M, Jeffriess L, Leano R, Mundy J, Marwick TH: Assessment of myocardial viability at dobutamine echocardiography by deformation analysis using tissue velocity and speckle-tracking. JACC CardiovasC Imaging 2010, 3(2):121-131

24. Roes SD, Mollema SA, Lamb HJ, van der Wall EE, de Roos A, Bax JJ: Validation of echocardiographic two-dimensional speckle tracking longitudinal strain imaging for viability assessment in patients with chronic ischemic left ventricular dysfunction and comparison with contrast-enhanced magnetic resonance imaging. Am J Cardiol 2009, 104 (3):312-317

25. Phan TT, Shivu GN, Abozguia K, Gnanadevan M, Ahmed I, Frenneaux M: Left ventricular torsion and strain patterns in heart failure with normal ejection fraction are similar to age-related changes. Eur J Echocardiogr 2009, 10(6):793-800.

26. Sjoli B, Orn S, Grenne B, Ihlen H, Edvardsen T, Brunvand H: Diagnostic capability and reproducibility of strain by Doppler and by speckle tracking in patients with acute myocardial infarction. JACC CardiovasC Imaging 2009, 2(1):24-33.

27. Kempny A, Diller GP, Orwat S, Kaleschke G, Kerckhoff G, Bunck AC, Maintz D, Baumgartner H: Right ventricular-left ventricular interaction in adults with Tetralogy of Fallot: A combined cardiac magnetic resonance and echocardiographic speckle tracking study. Int J Cardiol 2012, 154(3):259-264 
28. Van Dalen BM, Soliman OII, Vletter WB, Kauer F, Van Der Zwaan HB, Ten Cate FJ, Geleijnse ML: Feasibility and reproducibility of left ventricular rotation parameters measured by speckle tracking echocardiography. Eur J Echocardiogr 2009, 10(5):669-676.

29. Stanton T, Leano R, Marwick TH: Prediction of All-Cause Mortality From Global Longitudinal Speckle Strain: Comparison With Ejection Fraction and Wall Motion Scoring. Circ Cardiovasc Imaging 2009, 2(5):356-364.

doi:10.1186/1532-429X-14-43

Cite this article as: Morton et al:: Inter-study reproducibility of

cardiovascular magnetic resonance myocardial feature tracking. Journal of Cardiovascular Magnetic Resonance 2012 14:43.

\section{Submit your next manuscript to BioMed Central and take full advantage of:}

- Convenient online submission

- Thorough peer review

- No space constraints or color figure charges

- Immediate publication on acceptance

- Inclusion in PubMed, CAS, Scopus and Google Scholar

- Research which is freely available for redistribution 